\title{
ARTICLE Propofol decreases the excitability of cholinergic neurons in mouse basal forebrain via $\mathrm{GABA}_{\mathrm{A}}$ receptors
}

\author{
Lei Chen ${ }^{1}$, Zhi-lai Yang ${ }^{2}$, Juan Cheng ${ }^{1}$, Ping-ping Zhang ${ }^{1}$, Le-sha Zhang ${ }^{1}$, Xue-sheng Liu $^{2}$ and Lie-cheng Wang ${ }^{1}$
}

Propofol is an intravenous anesthetic that can active $\gamma$-aminobutyric acid $A\left(G A B A_{A}\right)$ receptors and generate sedative-hypnotic effects. Propofol has been widely applied clinically to achieve sedation comparable to sleep in humans. The basal forebrain (BF) is a brain region that plays an important role in sleep-wake regulation. Previous studies suggest that propofol affects the sleep-wake circuit via the BF; however, the mechanism remains elusive. In the current study we investigated the effects of propofol on the inherent properties of cholinergic neurons and their ability to convert excitatory inputs into spikes in mouse BF slices using wholecell patch clamp recordings. Bath application of propofol $(10 \mu \mathrm{M})$ significantly elevated the threshold potentials (Vts), decreased the number of spikes in response to a depolarizing current injection, and augmented the inter-spike intervals (ISIs), energy barrier (VtsVrs), and absolute refractory periods (ARPs). These effects were eliminated by co-application of a $\mathrm{GABA}_{\mathrm{A}}$ receptor antagonist picrotoxin $(50 \mu \mathrm{M})$. Altogether, our results reveal that propofol decreases the excitability of cholinergic neurons in mouse BF via $\mathrm{GABA}_{\mathrm{A}}$ receptors.

Keywords: propofol; basal forebrain; cholinergic neurons; picrotoxin; $\mathrm{GABA}_{\mathrm{A}}$ receptors; anesthesia

Acta Pharmacologica Sinica (2019) 40:755-761; https://doi.org/10.1038/s41401-018-0168-6

\section{INTRODUCTION}

Propofol has been widely used clinically as a short-acting anesthetic generating sedative-hypnotic effects, possibly by influencing the $\gamma$-aminobutyric acid $A\left(G A B A_{A}\right)$ [1-3], histamine $\mathrm{H}_{1}$, and histamine $\mathrm{H}_{2}$ receptors [4]. The most accepted anesthetic mechanism of propofol mainly relates to sleep-wake control, as the anesthetic state is comparable to sleep. Previous studies have considered the involvement of propofol on GABAergic neurons of the cerebral cortex $[5,6]$, hippocampus $[7,8]$, or ventrolateral preoptic nucleus [9-11]. However, the influence of propofol on cholinergic neurons of the basal forebrain (BF) remains elusive.

The BF is a large, heterogeneous structure located close to the ventral surface of the rostral telencephalon, and it is mainly involved in sleep-wake control [12-14]. In addition, it contains diverse neuronal types, including cholinergic, glutamatergic, parvalbumin-positive $\left(\mathrm{PV}^{+}\right)$GABAergic and somatostatin-positive $\left(\mathrm{SOM}^{+}\right)$GABAergic neurons [15]. The cholinergic, glutamatergic and $\mathrm{PV}^{+} \mathrm{GABAergic}$ neurons are wakefulness-promoting neurons $[16,17]$, while the $\mathrm{SOM}^{+}$GABAergic neurons are sleep-promoting neurons $[18,19]$.

The cholinergic neurons in the BF are significantly different from the GABAergic and glutamatergic neurons. A previous study used whole-cell recordings in acute slices and retrospective choline acetyltransferase (ChAT) immunocytochemistry to identify cholinergic neurons, and the researchers found that cholinergic neurons were mostly large neurons $(>20 \mu \mathrm{m})$ compared with the other two cell types $[20,21]$. The subthreshold characteristics of $\mathrm{ChAT}^{+}$and $\mathrm{ChAT}^{-}$neurons are similar. However, $\mathrm{ChAT}^{+}$neurons fire at lower frequencies than $\mathrm{ChAT}^{-}$neurons do during tonic depolarization [22-24].

The cholinergic dysfunction in the basal forebrain potentiates the anesthetic effects of propofol [25]. The local connectivity between different $\mathrm{BF}$ cell types indicates that the sleeppromoting somatostatin-positive $\left(\mathrm{SOM}^{+}\right)$GABAergic neurons inhibit the cholinergic neurons [13]. However, changes in BF cholinergic neurons' inherent properties and ability to convert excitatory inputs into spikes have not been assessed. Therefore, we hypothesized that propofol could change the excitabilities of cholinergic neurons in the $B F$, partly via $G_{A B A_{A}}$ receptors.

\section{MATERIALS AND METHODS}

Propofol (AstraZeneca, London, UK) was diluted with extracellular bathing solution to a final concentration of $10 \mu \mathrm{M}$ immediately before the experiments $[26,27]$. All other drugs (Sigma-Aldrich, St. Louis, USA) and all solutions (Sigma-Aldrich, St. Louis, USA) containing drugs were applied using a perfusion system (Longer Precision Pump Co., Ltd, Hebei, China) driven by gravitation.

BF slices and identification of cholinergic neurons

C57BL/6 mice weighing 16-18 g were housed under a $12 \mathrm{~h}$ light/ dark cycle (lights on at 6:00 a.m.). Food and water were available ad libitum. All experiments were approved by the Administration Office of Laboratory Animals of Anhui Medical University and

\footnotetext{
${ }^{1}$ Department of Pharmacology and Physiology, School of Basic Medical Sciences, Anhui Medical University, Hefei 230032, China and ${ }^{2}$ Department of Anesthesiology, First Affiliated Hospital of Anhui Medical University, Hefei 230022, China

Correspondence: Xue-sheng Liu (liu711029@hotmail.com) or Lie-cheng Wang (wangliecheng@ahmu.edu.cn)

These authors contributed equally: Lei Chen, Zhi-lai Yang
}

Received: 13 March 2018 Accepted: 31 August 2018

Published online: 26 October 2018 

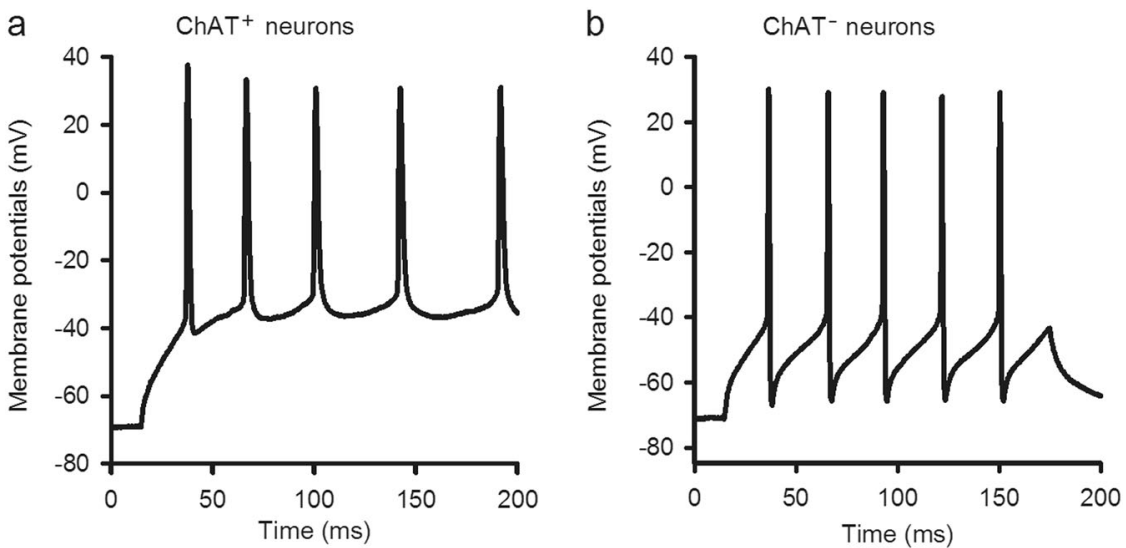

Fig. 1 Typical action potential firing of choline acetyltransferase-positive (ChAT ${ }^{+}$) neurons and choline acetyltransferase-negative (ChAT) neurons in the basal forebrain (BF) under whole-cell recordings. The spikes were induced by stimuli that were the same in intensity and duration under current-clamp recordings on $\mathrm{ChAT}^{+}$neurons and ChAT neurons in the BF. a The recorded spike trace illustrates the electrophysiologic properties of a ChAT ${ }^{+}$neuron. $\mathbf{b}$ The recorded spike trace illustrates the electrophysiologic properties of a ChAT neuron

adhered to the guidelines of the Institutional Animal Care Unit Committee of Anhui Medical University.

BF slices ( $400 \mu \mathrm{m}$ thick) were prepared from the C57BL/6 mice at 28 days after birth. All the mice were anesthetized by inhaling isoflurane and decapitated using a guillotine. The slices were cut using a vibratome (Leica Biosystems Inc. Buffalo Grove, United States) in oxygenated $\left(95 \% \mathrm{O}_{2} / 5 \% \mathrm{CO}_{2}\right)$ artificial cerebrospinal fluid (ACSF) containing (mM) $124 \mathrm{NaCl}, 3 \mathrm{KCl}, 1.2 \mathrm{NaH}_{2} \mathrm{PO}_{4}, 26$ $\mathrm{NaHCO}_{3}, 0.5 \mathrm{CaCl}_{2}, 4 \mathrm{MgSO}_{4}, 10$ dextrose, and 5 HEPES, pH 7.35, at $4{ }^{\circ} \mathrm{C}$. Then, the slices were moved to oxygenated ACSF containing (mM) $124 \mathrm{NaCl}, 3 \mathrm{KCl}, 1.2 \mathrm{NaH}_{2} \mathrm{PO}_{4}, 26 \mathrm{NaHCO}_{3}, 2.4 \mathrm{CaCl}_{2}, 1.3$ $\mathrm{MgSO}_{4}, 10$ dextrose, and 5 HEPES, pH 7.35, at $25^{\circ} \mathrm{C}$ for $1 \mathrm{~h} \mathrm{[28].}$ The slices were transferred to a submersion chamber perfused with the oxygenated ACSF at $30^{\circ} \mathrm{C}$ for whole-cell patch clamp recordings.

In the preparation of slices, we selected the slice next to the anterior part of the anterior commissure (ACA) and the posterior part of the anterior commissure (ACP) because the ACA and ACP are clearly observed in the slice. Meanwhile, only one cell was recorded in each slice of $B F$ because of the long wash-out time. Propofol $(10 \mu \mathrm{M})$ was added to the ACSF to examine whether the inherent properties of the cholinergic neurons were changed. Picrotoxin (PTX, $50 \mu \mathrm{M})$ and propofol $(10 \mu \mathrm{M})$ were added to the $A C S F$ at the end of the experiment to examine whether $G_{A B A}$ receptors were involved in this function. Electrophysiological recordings on the neurons in BF were conducted under a microscope (Olympus Corporation, Tokyo, Japan). The cholinergic neurons were larger than the other neurons, and most were in the substantia innominata (SI) of the BF.

Whole-cell patch clamp recordings

The neurons were recorded using a MultiClamp-700B amplifier (Molecular Device, San Jose, CA, USA) in current-clamp mode. Electrical signals were recorded using the pClamp-10 software (Axon Instrument Inc., San Jose, CA, USA) for data acquisition and analysis. The patch clamp recordings were sampled at $50 \mathrm{kHz}$ and low-pass-filtered at $10 \mathrm{kHz}$. The pipette solution for recording the spikes contained (in mM) 150 K-gluconate, $5 \mathrm{NaCl}, 5$ HEPES, 0.4 EGTA, 4 Mg-ATP, 0.5 Tris-GTP, and 5 phosphocreatine (pH 7.35) [28]. The pipette solutions were freshly made and filtered $(0.1 \mathrm{~mm})$ before use. The osmolarity was 295-305 mOsmol, and the pipette resistance was 5-6 M $\Omega$.

The action potentials of the BF neurons were induced by injecting depolarization pulses. The ability to convert excitatory inputs into digital spikes was evaluated by the input-output relationship when a series of stimuli with gradually increasing intensity were given, as well as by the inter-spike intervals (ISIs) when the same stimulus intensity and duration were given. The neural-intrinsic properties of the threshold potentials (Vts), resting potentials (Vrs), and absolute refractory periods (ARPs) were used to evaluate the neuronal excitability. We did not measure the rheobase to show neuronal excitability, as this strength-duration relationship is used to indicate the ability to fire a single spike; the ability to produce sequential spikes was measured in the current study. The energy barrier (Vts-Vrs), which is the difference of the membrane potential between Vrs and Vts, was demonstrated by injecting a threshold stimulus. ARPs were measured by injecting paired-depolarization pulses ( $3 \mathrm{~ms}$, four times higher than the threshold intensity) into the neurons, with a specific interpulse duration, after each spike. By changing the inter-pulse intervals, the ARPs were defined as the time from a complete spike to the next spike at $50 \%$ probability. We first recorded ISIs, Vts, Vrs, and APRs before the drug application (approximately $10 \mathrm{~min}$ ). The four indexes were recorded again 5 min after $10 \mu \mathrm{M}$ propofol or $50 \mu \mathrm{M}$ PTX +10 $\mu \mathrm{M}$ propofol application $[29,30]$.

The data were analyzed if the recorded neurons exhibited negative resting membrane potentials more than $-60 \mathrm{mV}$. The criteria for the acceptance of each experiment also included less than $5 \%$ changes in the resting membrane potential, spike magnitude, and input resistance throughout the experiment. The series and input resistances in all the neurons were monitored by injecting hyperpolarization pulses $(5 \mathrm{mV} / 50 \mathrm{~ms})$ and were calculated by the voltage pulses vs. instantaneous and steady-state currents. To estimate the effects of propofol on neuronal spikes, the energy barrier, ISIs, input-output, and ARPs, neurons in the slices were measured before and after propofol administration.

Statistical analyses

The data from the electrophysiological recordings are presented as the mean \pm standard error of the mean. The paired $t$-test was used in the statistical comparisons of the experimental data before and after administering propofol in the threshold stimulus intensity and absolute refractory periods. Repeated-measures oneway ANOVA with post hoc comparisons was used to make statistical comparisons in the input-output, ISIs and energy barrier between before and after drug administration. All data were confirmed to be normally distributed with equal variances before statistical comparisons. 

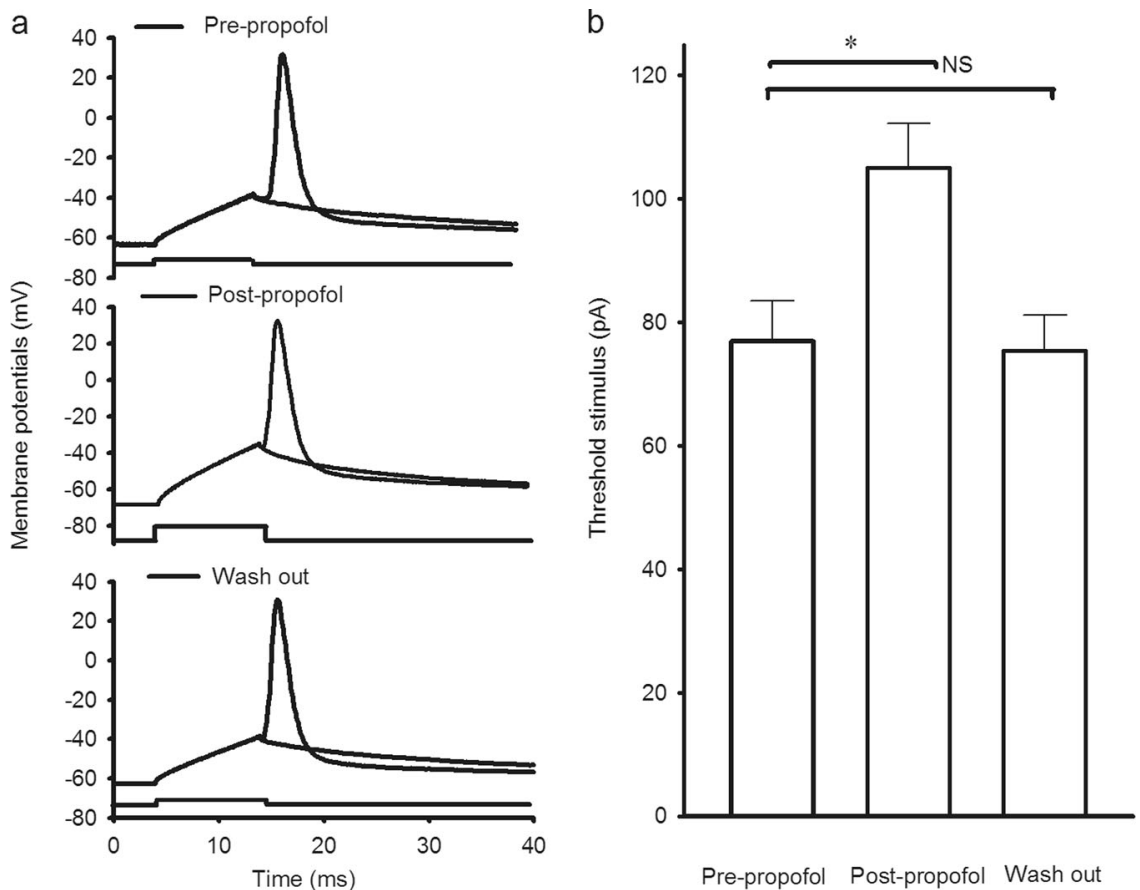

Fig. 2 Threshold stimulus intensity in the cholinergic neurons in the BF was increased after propofol administration. The spikes were induced by the threshold stimulus intensity under current-clamp recordings in cholinergic neurons in the BF. a Recorded traces illustrate the threshold stimulus-induced action potential pre-propofol (upper), post-propofol (middle) and during washout (lower) in a cholinergic neuron. b Quantification of the data shows the threshold stimulus intensity of the action potential pre-propofol, post-propofol administration and during washout $\left({ }^{*} P<0.05\right.$, pre-propofol vs. post-propofol, $n=10$ neurons from 10 mice; NS means $P>0.05$, post-propofol vs. wash out, $n=5$ neurons from 5 mice). Mean \pm SEM

a
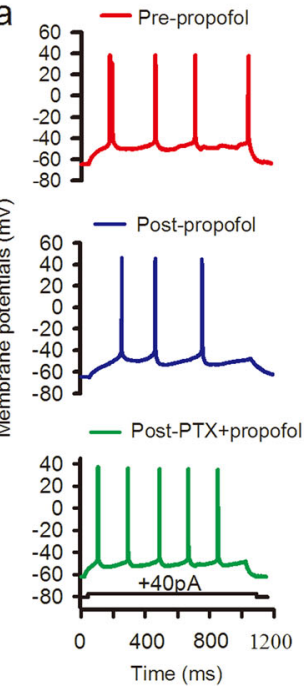
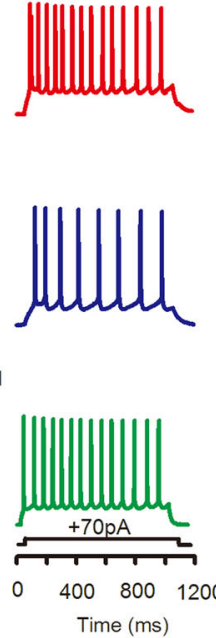
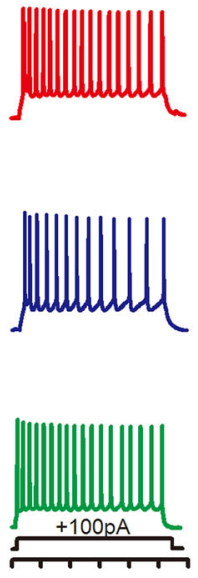

$400800 \quad 1200$ b

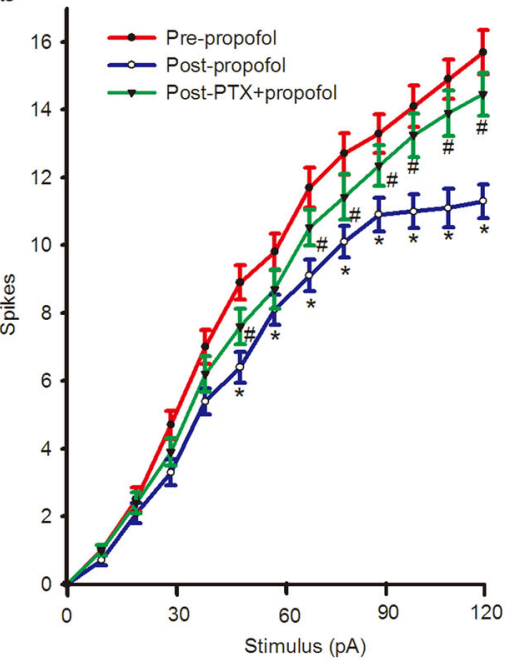

Fig. 3 Coding abilities were decreased in cholinergic neurons in the BF after propofol administration, which were eliminated by PTX. a Sequential spikes were induced by a series of stimuli that were gradually increased in intensity under current-clamp recordings in BF cholinergic neurons. Groups of typical sequential spikes in response to a depolarization pulse with the same intensity are shown from top to bottom, under the conditions of pre-propofol (red trace), post-propofol (blue trace) and post-PTX + propofol (green trace) in a cholinergic neuron. $\mathbf{b}$ Quantification of the data shows the numbers of spikes with different stimuli was significantly decreased under the conditions of post-propofol compared with pre-propofol ( ${ }^{*} P<0.05$, prepropofol vs. post-propofol; $n=10$ neurons from 10 mice), and this effect was eliminated by PTX ( ${ }^{\#} P<0.05$, post-PTX + propofol vs. post-propofol; $n=9$ neurons from 9 mice). Mean \pm SEM

RESULTS

The basic property of action potential firing in $\mathrm{ChAT}^{+}$and $\mathrm{ChAT}^{-}$ neurons

Previous studies [20-24] have demonstrated the electrophysiological properties of cholinergic and noncholinergic neurons in the
BF. The action potentials of $\mathrm{ChAT}^{+}$and $\mathrm{ChAT}^{-}$neurons have different characteristics in whole-cell patch clamp recordings. Here, the action potential firing characteristics of $\mathrm{ChAT}^{+}$neurons and ChAT neurons were recorded using the same stimulus (Fig. 1) [22]. The $\mathrm{ChAT}^{+}$neurons were magnocellular neurons, and they 

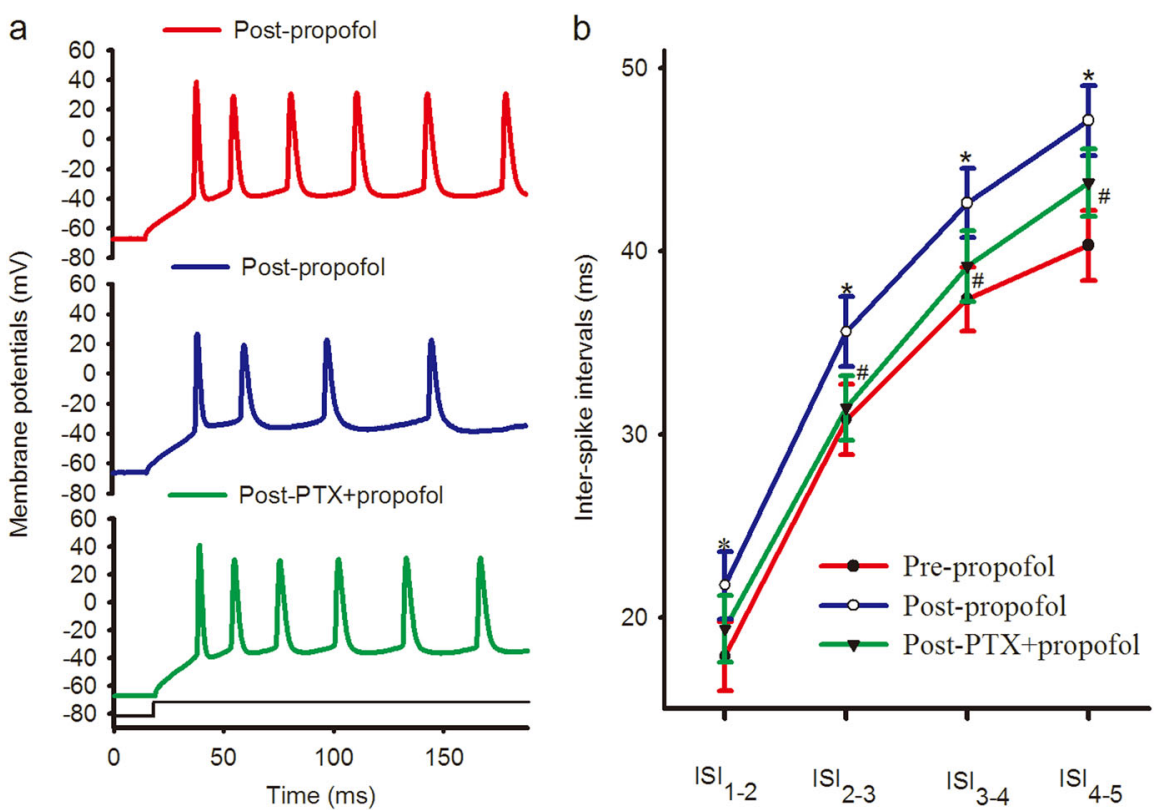

Fig. 4 Inter-spike intervals (ISIs) were enlarged in cholinergic neurons in the BF after propofol administration, which were eliminated by PTX. a The spikes were induced by stimuli with the same intensity and duration under current-clamp recordings in cholinergic neurons of the BF. Traces illustrate the depolarization-induced spikes pre-propofol (red), post-propofol (blue) and post-PTX + propofol (green) in a cholinergic neuron. $\mathbf{b}$ Quantification of the data shows that the ISIs of spikes 1-2 to 4-5 were significantly prolonged in the conditions of post-propofol compared with pre-propofol ( ${ }^{*} P<0.05$, pre-propofol vs. postpropofol; $n=10$ neurons from 10 mice), and this effect was eliminated by PTX ( ${ }^{\#} P<0.05$, post-PTX + propofol vs. post-propofol; $n=9$ neurons from 9 mice). Mean \pm SEM

fired at lower frequencies than the ChAT neurons stimulated by tonic depolarization.

Enhancement of threshold stimulus intensity of BF cholinergic neurons by propofol

The intensity of the injected depolarization pulse that just induced an action potential was called the threshold stimulus intensity (Fig. 2a). Compared with the pre-propofol group, the threshold stimulus intensity of the $\mathrm{BF} \mathrm{ChAT}^{+}$neurons was increased by approximately $35 \%$ in the post-propofol group. The threshold stimulus intensity was $77.01 \pm 6.54 \mathrm{pA}$ in the pre-propofol group and $105.01 \pm 7.15 \mathrm{pA}$ in the post-propofol group $(P<0.05$, prepropofol compared with post-propofol; $n=10$ neurons from 10 mice; Fig. 2b). At the end of the experiment, the threshold stimulus intensity recovered to the pre-propofol level $(P>0.05$, pre-propofol compared with wash out; $n=5$ neurons from 5 mice; Fig. 2b). Moreover, there was no significant difference in the Vrs before and after propofol administration $(P>0.05$, pre-propofol: $64.75 \pm 1.81 \mathrm{mV}$; Post-propofol: $65.28 \pm 1.93 \mathrm{mV} ; n=10$ neurons from 10 mice). These results indicate that the threshold stimulus intensity for BF cholinergic neurons was enhanced after propofol administration.

Downregulation of the excitability of BF cholinergic neurons by propofol

A previous study [25] indicated that cholinergic neurons were involved in anesthesia function by intraperitoneal administration of propofol in rats. However, the cellular mechanism of this effect is still unknown. Here, we analyzed the input-output curve and ISIs of cholinergic neurons in the BF before and after propofol administration.

Cholinergic neurons' ability to convert excitatory inputs into digital spikes was demonstrated by a series of stimuli with gradually increased intensity under current-clamp recordings (Fig. 3a). The numbers of spikes increased according to the increasing intensity of the stimulus. Nevertheless, propofol significantly decreased the number of spikes compared with the pre-propofol results under the same stimulus intensity $(P<0.05$, pre-propofol compared with post-propofol; $n=10$ neurons from 10 mice; Fig. 3b). The input-output curve for the pre-propofol neurons shifted right and down after propofol administration.

The ISIs, which represented the time interval between two neighboring spikes under the threshold stimulus intensity, were also measured. Spiking traces in cholinergic neurons of the BF from pre-propofol (red) and post-propofol (blue) groups, respectively, were induced by stimuli with the same intensity and duration (Fig. 4a). The ISIs values of spikes 1-2, 2-3, 3-4, and 4-5 were $17.86 \pm 1.89,30.80 \pm 1.91,37.38 \pm 1.74$, and $40.33 \pm 1.91 \mathrm{~ms}$, respectively, in the pre-propofol group and $21.75 \pm 1.84,35.60 \pm$ $1.90,42.64 \pm 1.89$, and $47.14 \pm 1.92 \mathrm{~ms}$ in the post-propofol group $(P<0.05$, pre-propofol compared with post-propofol; $n=10$ neurons from 10 mice; Fig. 4b). Thus, the ISIs was significantly prolonged after propofol administration. This indicated that propofol downregulated the ability of BF cholinergic neurons to convert the excitatory inputs into digital spikes.

The excitability of the cholinergic neurons was also evaluated by the ARPs and the energy barrier, which reflected the characteristics of voltage-gated sodium channels. The ARPs were measured by injecting paired-depolarization pulses $(3 \mathrm{~ms}$, four times higher than the threshold intensity) with a specific interpulse interval under current-clamp recordings, which generated two action potentials. By changing the interpulse intervals, the ARPs were defined as the time latency when the subsequent spike was at $50 \%$ probability (Fig. 5a). The ARP values of the spikes were $6.44 \pm 0.59 \mathrm{~ms}$ in the pre-propofol group and $7.1 \pm 0.66 \mathrm{~ms}$ in the post-propofol group $(P<0.05$, pre-propofol compared with post-propofol; $n=10$ neurons from 10 mice; Fig. 5b).

The energy barrier, which was the difference in the membrane potential from Vrs to Vts, was demonstrated by injecting a threshold stimulus (Fig. 6a). The energy barrier values of spikes $1-4$ were $25.21 \pm 0.80,28.37 \pm 0.85,29.62 \pm 0.78$, and $30.20 \pm$ $0.80 \mathrm{mV}$, respectively, in the pre-propofol group and $27.58 \pm$ $0.79,30.25 \pm 0.81,31.15 \pm 0.79$, and $31.78 \pm 0.84 \mathrm{mV}$ in the post-propofol group $(P<0.05$, pre-propofol compared with 
a

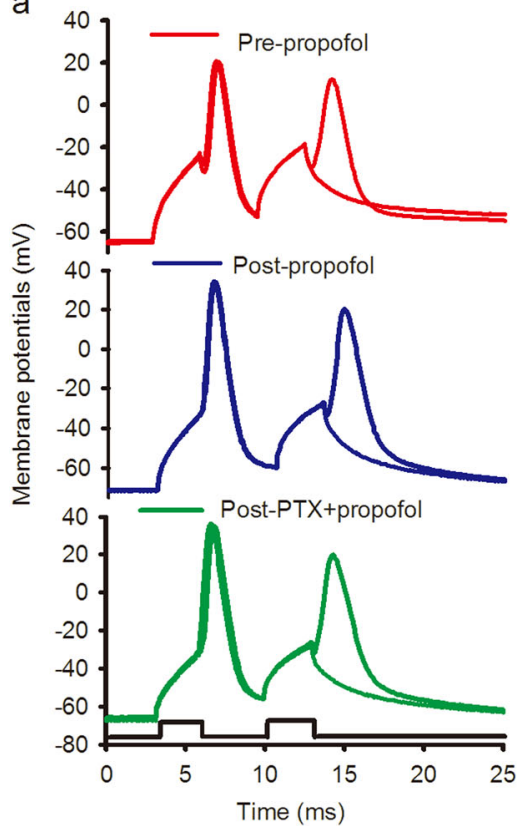

b

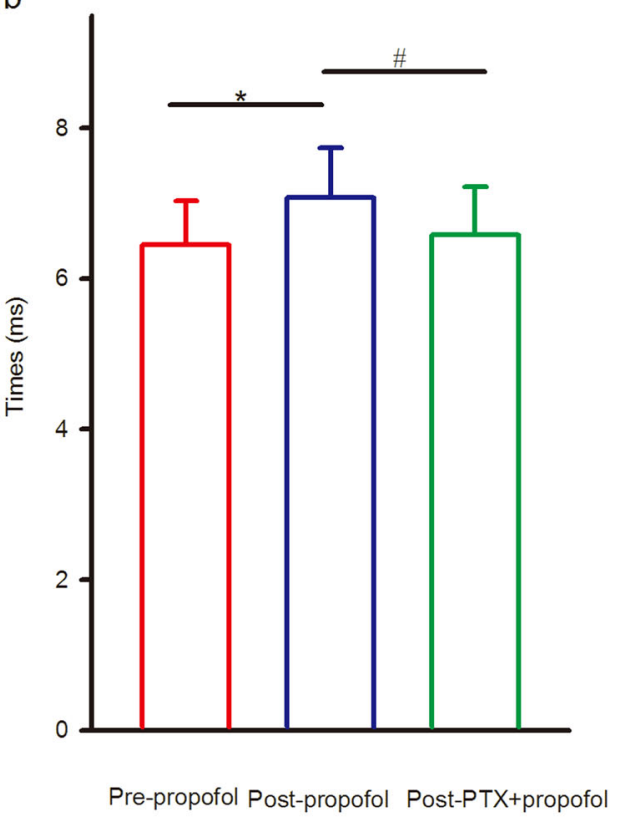

Fig. 5 Absolute refractory period (ARP) was prolonged in cholinergic neurons in the BF after propofol administration, which was eliminated by PTX. a The spikes were induced by injecting paired pulse stimuli ( $3 \mathrm{~ms}$, four times higher than the threshold intensity) under current-clamp recordings in cholinergic neurons of the BF. Traces illustrate paired depolarization-induced spikes pre-propofol (red), post-propofol (blue) and post-PTX + propofol (green) in a cholinergic neuron (note the different durations between the two spikes in the three groups). b Quantification of the data shows the spikes' ARPs were significantly enlarged postpropofol compared with pre-propofol $(* P<0.05$, prepropofol vs. post-propofol; $n=10$ neurons from 10 mice), and this effect was eliminated by PTX ( $P<0.05$, post-PTX + propofol vs. postpropofol; $n=9$ neurons from 9 mice). Mean \pm SEM

post-propofol; $n=10$ neurons from 10 mice; Fig. 6b). Both the ARPs and energy barriers of the spikes were significantly enhanced after propofol administration. This indicated that propofol might downregulate the excitability of BF cholinergic neurons.

Involvement of $\mathrm{GABA}_{\mathrm{A}}$ receptors in the decreased excitability of $\mathrm{BF}$ cholinergic neurons by propofol

Propofol is believed to exert its anesthetic effect through $G_{A B A_{A}}$ receptors [1-3]. Therefore, the $\mathrm{GABA}_{A}$ receptor antagonist PTX was used to examine the role of this family of receptors. The shifting of the input-output curve evoked by propofol was reversed by PTX $(P<0.05$, post-propofol compared with post-PTX + propofol; $n=9$ neurons from 9 mice; green in Fig. 3). The increment of ISIs by propofol was also reversed by PTX (the values of ISIs between spikes $1-2,2-3,3-4$, and $4-5$ were $21.75 \pm 1.84,35.60 \pm 1.90$, $42.64 \pm 1.89$, and $47.14 \pm 1.92 \mathrm{~ms}$ in the post-propofol group, and $19.37 \pm 1.83,31.42 \pm 1.75,39.17 \pm 1.93$, and $43.75 \pm 1.84 \mathrm{~ms}$ in the post-PTX + propofol group, $P<0.05$, post-propofol compared with post-PTX + propofol; $n=9$ neurons from 9 mice; green in Fig. 4). PTX also reversed ARP prolongation by propofol $(7.1 \pm 0.66 \mathrm{~ms}$ in the post-propofol group and $6.58 \pm 0.63 \mathrm{~ms}$ in the post-PTX + propofol group, $P<0.05$, post-propofol compared with post-PTX + propofol; $n=9$ neurons from 9 mice; green in Fig. 5). Similarly, PTX reversed the increased energy barrier by propofol (the values of energy barrier of spikes $1-4$ were $27.58 \pm 0.79$, $30.25 \pm 0.81,31.15 \pm 0.79$, and $31.78 \pm 0.84 \mathrm{mV}$ in the postpropofol group and $25.90 \pm 0.67,28.88 \pm 0.72,30.12 \pm 0.81$, and $30.57 \pm 0.78 \mathrm{mV}$ in the post-PTX + propofol group, $P<0.05$, postpropofol compared with post-PTX + propofol; $n=9$ neurons from 9 mice; green in Fig. 6). We also examined whether propofol was still effective in the presence of PTX. As a result, propofol had no effect on cholinergic neurons of the BF in the presence of PTX (data not shown).

\section{DISCUSSION}

Sleep and general anesthesia might share some similar neural pathways. Certain brain areas involved in sleep also play important roles in general anesthesia [31-33]. Propofol is an intravenous anesthetic that has been widely applied clinically to sedate patients. The BF is mainly involved in sleep-wake control, and the cholinergic neurons in BF are wakefulness-promoting neurons. Despite its worldwide application in clinical trials, the mechanism of propofol has still not been clearly clarified.

Electrophysiological studies suggest that the features of slow waves are similar between non-REM sleep and sedation under propofol [34, 35]. The active area of propofol is associated with the brain nucleus and neural circuits of sleep [36]. Ventrolateral preoptic nucleus (VLPO) is a sleep-promoting center. Noradrenalin-inhibited (NA-) GABAergic neurons in VLPO promote sleep, and propofol can activate NA (-) neurons by inhibiting GABAergic transmission [36]. NA (-) neurons in VLPO and cholinergic neurons in BF also play important roles in wake-sleep control. The cholinergic neurons are distributed throughout the BF and mainly arise from the SI of the BF [37]. Many ChAT neurons located in the rostral $B F$ maintain wakefulness, while the other ChAT neurons, located in the caudal region of the BF, innervate the suprachiasmatic nucleus, the circadian pacemaker [38, 39]. In our study, the neurons were mainly selected from these two regions. Recently, a whole-brain atlas of the cholinergic system was completed, clarifying the distribution of cholinergic neurons in the brain [40]. In the local wake-sleep circuit of BF, the role of cholinergic neurons and the $\mathrm{SOM}^{+}$GABAergic neurons is bidirectional [41]. Activation of $\mathrm{SOM}^{+}$neurons may facilitate spontaneous transitions into non-REM sleep by direct postsynaptic inhibition of wakepromoting cholinergic neurons.

Propofol exerts its sedative effect via $G_{A B A}$ receptors. PTX, a competitive $G_{A B A_{A}}$ receptor antagonist, can block both the phasic current and the tonic currents. Clinically relevant concentrations of propofol induce a greater increase in tonic currents [42]. 

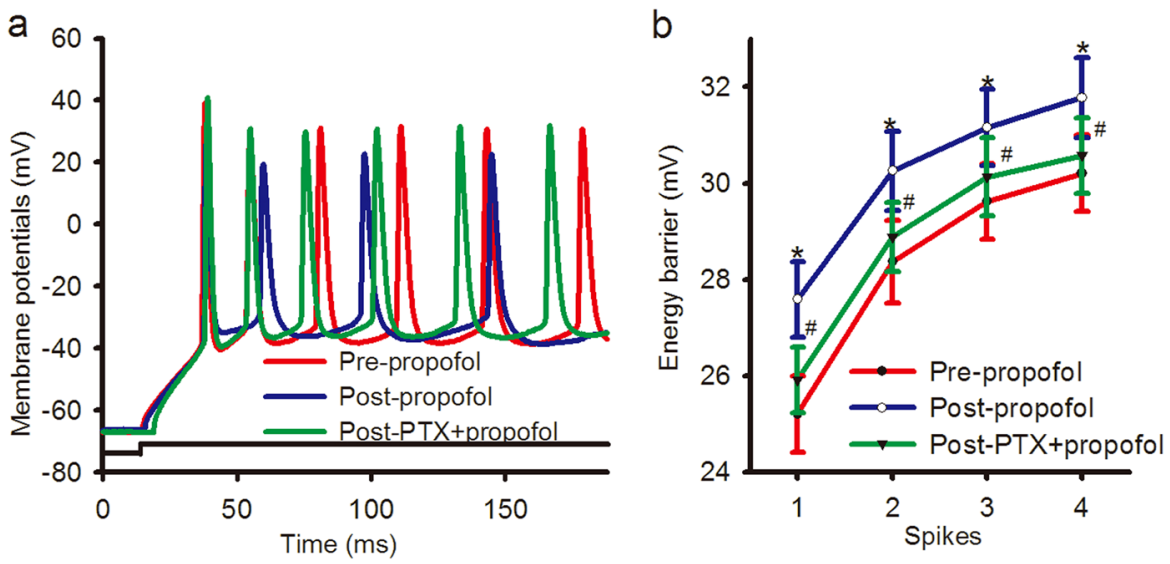

Fig. 6 Energy barrier was increased in cholinergic neurons in the BF after propofol administration, which was eliminated by PTX. a The spikes were induced by stimuli of the same intensity and duration under current-clamp recordings in cholinergic neurons of the BF. Traces illustrate depolarization-induced spikes pre-propofol (red), post-propofol (blue) and post-PTX + propofol (green) in a cholinergic neuron. b Quantification of the data show that the energy barrier of the spikes was significantly enhanced postpropofol compared with pre-propofol ( ${ }^{*} P<0.05$, pre-propofol vs. post-propofol; $n=10$ neurons from 10 mice), and this effect was eliminated by PTX ( ${ }^{\#}<<0.05$, post-PTX + propofol vs. post-propofol; $n=9$ neurons from 9 mice). Mean \pm SEM

The tonic GABA current is different from the GABA current of quantal synaptic transmission because receptors underlying tonic activation may be different from receptors underlying phasic activation. Drugs that potentiate the functions of $\mathrm{GABA}_{\mathrm{A}}$ receptors, for example, the benzodiazepines, also increase the tonic GABA currents, but the amplitude enhancement of tonic currents is different $[43,44]$.

Here, we report the influence of propofol on the inherent excitatory properties of BF cholinergic neurons by patch clamp recordings. We recorded the input-output curve, ISIs, ARPs and energy barrier of BF cholinergic neurons and found the downregulation of the inherent excitatory properties after propofol application. Hyperpolarization of the neurons can affect the inherent excitatory properties. In order to exclude the influence of the Vrs, we analyzed the Vrs and found no significant difference in the Vrs before and after propofol administration. Additionally, those effects were eliminated by PTX, a $G_{A B A}$ receptor antagonist. To our knowledge, this is the first exploration of the influence of propofol on the inherent excitability of cholinergic neurons. In brief, propofol can decrease the excitability of cholinergic neurons via $G_{A B A}$ receptors.

\section{ACKNOWLEDGEMENTS}

We thank Dr. Zhi-gang XIONG for critical comments on the manuscript. This study was supported by the National Natural Science Foundation of China (81571293 to LcW; 81500949 to Z-IY; 81571039 to X-sL) and the National Century Excellent Talents in University of China (gxbjZD2016034 to L-cW).

\section{AUTHOR CONTRIBUTIONS}

$X-s L$ and L-cW designed the research; LC and Z-IY mainly performed the research; JC, P-pZ and L-sZ partly performed the research and analyzed the data; LC and L-cW analyzed the data and wrote the paper.

\section{ADDITIONAL INFORMATION}

Competing interests: The authors declare no competing interests.

\section{REFERENCES}

1. Bali $M$, Akabas MH. Defining the propofol binding site location on the GABAA receptor. Mol Pharmacol. 2004;65:68-76.

2. Ishiguro M, Kobayashi S, Matsuyama K, Nagamine T. Effects of propofol on IPSCS in CA1 and dentate gyrus cells of rat hippocampus: propofol effects on hippocampal cells' IPSCs. Neurosci Res. 2018;17:30716-2.
3. Jayakar SS, Zhou X, Chiara DC, Dostalova Z, Savechenkov PY, Bruzik KS, et al. Multiple propofol-bingding sites in a $\gamma$-aminobutyric acid type $A$ receptor (GABAAR) identified using a photoreactive propofol analog. J Bioll Chem. 2014; 289:27456-68

4. Liu Y, Zhang Y, Qian $\mathrm{K}$, Zhang L, Yu T. Histaminergic $\mathrm{H} 1$ and $\mathrm{H} 2$ receptors mediate the effects of propofol on the noradrenalin-inhibited neurons in rat ventrolateral preoptic nucleus. Neurochem Res. 2017;42:1387-93.

5. Kobayashi M, Oi Y. Actions of propofol on neurons in the cerebral cortex. J Nippon Med Sch. 2017;84:165-9.

6. Briner Adrian, Nikonenko Irina, Roo MathiasDe, Dayer Alexandre, Muller Dominique. Developmental stage-dependent persistent impact of propofol anesthesia on dendritic spines in the rat medial prefrontal cortex. Anesthesiology. 2011; 115:282-93.

7. Xie F, Li X, Bao M, Shi R, Guan Y, Wang Y. Anesthetic propofol normalized the increased release of glutamate and $Y$-amino butyric acid in hippocampus after paradoxical sleep deprivation in rats. Neurol Res. 2015;37:1102-7.

8. Feng CS, Qiu JP, Ma HC, Yue Y. Effect of propofol on synaptic long-term potentiation in hippocampal slices of rats. Zhonghua Yi Xue Za Zhi. 2007; 87:763-7.

9. Zhang Y, Yu T, Yuan J, Yu BW. The ventrolateral preoptic nucleus is required for propofol-induced inhibition of locus coeruleus neuronal activity. Neurol Sci. 2015;36:2177-84.

10. Li KY, Guan YZ, Krnjevic K, Ye JH. Propofol facilitates glutamatergic transmission to neurons of the ventrolateral preoptic nucleus. Anesthesiology. 2009; 111:1271-8.

11. Zhang Y, Yu T, Liu Y, Qian K, Yu BW. Muscarinic M1 receptors regulate propofol modulation of GABAergic transmission in rat ventrolateral preoptic neurons. J Mol Neurosci. 2015;55:830-5.

12. Do JP, Xu M, Lee SH, Chang WC, Zhang $S$, Chung $S$, et al. Cell type-specific longrange connections of basal forebrain circuit. eLife. 2016;19:1-17.

13. Xu M, Chung S, Zhang S, Zhong P, Ma C, Chang WC, et al. Basal forebrain circuit for sleep-wake control. Nat Neurosci. 2015;18:1641-47.

14. Anaclet C, Pedersen NP, Ferrari LL, Venner A, Bass CE, Arrigoni E, et al. Basal forebrain control of wakefulness and cortical rhythms. Nat Commun. 2015; 6:8744.

15. Yang C, Thankachan S, McCarley RW, Brown RE. The menagerie of the basal forebrain: how many (neural) species are there, what do they look like, how do they behave and who talks to whom? Curr Opin Neurobiol. 2017;44:159-66.

16. Zant JC, Kin T, Prokai L, Szarka S, McNally J, McKenna JT, et al. Cholinergic neurons in the basal forebrain promote wakefulness by actions on neighboring non-cholinergic neurons: an opto-dialysis study. J Neurosci. 2016;36:2057-67.

17. Shi YF, Yu YQ. The roles of glutamate in sleep and wakefulness. Zhejiang Da Xue Xue Bao Yi Xue Ban. 2013;42:583-90.

18. Anaclet C, Ferrari L, Arrigoni E, Bass CE, Saper CB, Lu J, et al. The GABAergic parafacial zone is a medullary slow wave sleep-promoting center. Nat Neurosci. 2014;17:1217-24.

19. Yuan J, Luo Z, Zhang Y, Zhang Y, Wang Y, Cao S, et al. GABAergic ventrolateral pre-optic nucleus neurons are involved in the mediation of the anesthetic hypnosis induced by propofol. Mol Med Rep. 2017;16:3179-86. 
20. Serafin $M$, Williams $S$, Khateb A, Fort $P$, Mühlethaler $M$. Rhythmic firing of medial septum non-cholinergic neurons. Neuroscience. 1996;75:671-5.

21. Markram H, Segal M. Electrophysiological characteristics of cholinergic and noncholinergic neurons in the rat medial septum-diagonal band complex. Brain Res. 1990;513:171-4.

22. Hedrick T, Water J. Physiological properties of cholinergic and non-cholinergic magnocellular neurons in acute slices from adult mouse nucleus basalis. PLoS One. 2010;5:1-9.

23. Unal CT, Golowasch JP, Zaborszky L. Adult mouse basal forebrain harbors two distinct cholinergic populations defined by their electrophysiology. Front Behav Neurosci. 2012;6:1-14

24. López-Hernández GY, Ananth M, Jiang L, Ballinger EC, Talmage DA, Role LW. Electrophysiological properties of basal forebrain cholinergic neurons identified by genetic and optogenetic tagging. J Neurochem. 2017;142:103-10.

25. Laalou FZ, de Vasconcelos AP, Oberling P, Jeltsch H, Cassel JC, Pain L. Involvement of the basal cholinergic forebrain in the mediation of general (propofol) anesthesia. Anesthesiology. 2008;108:888-96.

26. Jin Z, Choi MJ, Park CS, Park YS, Jin YH. Propofol facilitated excitatory postsynaptic currents frequency on nucleus tractus solitarii (NTS) neurons. Brain Res. 2012;1432:1-6

27. Li KeY, Guan Yan-zhong, Krnjević Kresimir, Jiang, Ye H. Propofol facilitates glutamatergic transmission to neurons of the ventrolateral preoptic nucleus. Anesthesiology. 2009;111:1271-78.

28. Gao Z, Chen L, Fan R, Lu W, Wang D, Cui S, et al. Associations of unilateral whisker and olfactory signals induce synapse formation and memory cell recruitment in bilateral barrel cortices: cellular mechanism for unilateral training toward bilateral memory. Front Cell Neurosci. 2016;10:1-16.

29. Thomas P, Mortensen M, Hosie AM, Smart TG. Dynamic mobility of functional GABAA receptors at inhibitory synapses. Nat Neurosci. 2005;8:889-97.

30. Schummers J, Browning MD. Evidence for a role for GABAA and NMDA receptors in ethanol inhibition of long-term potentiation. Brain Res Mol Brain Res. 2001;94:9-14.

31. Franks NP. General anaesthesia: from molecular targets to neuronal pathways of sleep and arousal. Nat Rev Neurosci. 2008;9:370-86.

32. Zecharia Anna Y, Nelson Laura E, Gent Thomas C, Schumacher Mark, Jurd Rachel, Rudolph Uwe, et al. The involvement of hypothalamic sleep pathways in general anesthesia: testing the hypothesis using the $\mathrm{GABA}_{\mathrm{A}}$ receptor $33 \mathrm{~N} 265 \mathrm{M}$ knock-in mouse. J Neurosci. 2009;29:2177-87.
33. Nelson LE, Guo TZ, Lu J, Saper CB, Franks NP, Maze M. The sedative component of anesthesia is mediated by $\mathrm{GABA}_{\mathrm{A}}$ receptors in an endogenous sleep pathway. Nat Neurosci. 2002;5:979-84.

34. Li Y, Wang S, Pan C, Xue F, Xian J, Huang Y, et al. Comparison of NREM sleep and intravenous sedation through local information processing and whole brain network to explore the mechanism of general anesthesia. PLoS One. 2018;13: e0192358.

35. Murphy Michael, Bruno Marie-Aurélie, Riedner Brady A, Boveroux Pierre, Noirhomme Quentin, Landsness Eric C, et al. Propofol anesthesia and sleep: a highdensity EEG study. Sleep. 2011;34:283-91.

36. Liu YW, Zuo W, Ye JH. Propofol stimulates noradrenalin-inhibited neurons in the ventrolateral preoptic nucleus by reducing GABAergic inhibition. Anesth Analg. 2013;117:358-63.

37. Yang C, McKenna JT, Zant JC, Winston S, Basheer R, Brown RE. Cholinergic neurons excite cortically projecting basal forebrain GABAergic neurons. J Neurosci. 2014;34:2832-44.

38. Yamakawa GR, Basu P, Cortese F, MacDonnell J, Whalley D, Smith VM, et al. The cholinergic forebrain arousal system acts directly on the circadian pacemaker PNAS. 2016;113:13498-503.

39. Bina KG, Rusak B, Semba K. Localization of cholinergic neurons in the forebrain and brainstem that project to the suprachiasmatic nucleus of the hypothalamus in rat. J Comp Neurol. 1993;335:295-307.

40. Li X, Yu B, Sun Q, Zhang Y, Ren M, Zhang X, et al. Generation of a whole-brain atlas for the cholinergic system and mesoscopicprojectome analysis of basal forebrain cholinergic neurons. PNAS. 2018;115:415-20.

41. Donlea JM, Alam MN, Szymusiak R. Neuronal substrates of sleep homeostasis; lessons from flies, rats and mice. Curr Opin Neurobiol. 2017; 44:228-35.

42. Semyanov A, Walker MC, Kullmann DM. GABA uptake regulates cortical excitability via cell type-specific tonic inhibition. Nat Neurosci. 2003;6:484-90.

43. Bai D, Zhu G, Pennefather $P$, Jackson MF, MacDonald JF, Orser BA. Distinct functional and pharmacological properties of tonic and quantal inhibitory postsynaptic currents mediated by $\mathrm{Y}$-aminobutyric acid A receptors in hippocampal neurons. Mol Pharmacol. 2001;59:814-24.

44. Narimatsu E, Niiya T, Kawamata M, Namiki A. The mechanisms of depression by benzodiazepines, barbiturates and propofol of excitatory synaptic transmissions mediated by adenosine neuromodulation. Masui. 2006;55: 684-91. 